\title{
THE CONVERSE OF THE MINKOWSKI'S INEQUALITY THEOREM AND ITS GENERALIZATION
}

\author{
JANUSZ MATKOWSKI
}

(Communicated by R. Daniel Mauldin)

\begin{abstract}
Let $(\Omega, \Sigma, \mu)$ be a measure space with two sets $A, B \in \Sigma$ such that $0<\mu(A)<1<\mu(B)<\infty$, and let $\varphi$ : $\mathbf{R}_{+} \rightarrow \mathbf{R}_{+}$be bijective and $\varphi^{-1}$ continuous at 0 . We prove that if for all $\mu$-integrable step functions $x, y: \Omega \rightarrow \mathbf{R}$,

$$
\varphi^{-1}\left(\int_{\Omega} \varphi \circ|x+y| d \mu\right) \leq \varphi^{-1}\left(\int_{\Omega} \varphi \circ|x| d \mu\right)+\varphi^{-1}\left(\int_{\Omega} \varphi \circ|y| d \mu\right)
$$

then $\varphi(t)=\varphi(1) t^{p}$ for some $p \geq 1$. In the case of normalized measure we prove a generalization of Minkowski's inequality theorem. The suitable results for the reversed inequality are also presented.
\end{abstract}

\section{INTRODUCTION}

Let $(\Omega, \Sigma, \mu)$ be a measure space. The celebrated Minkowski's inequality theorem may be formulated in the form of the following implication.

$$
\text { If } \varphi(t)=\varphi(1) t^{p}(t \geq 0) \text {, where } p \geq 1 \text { and } \varphi(1)>0 \text {, then }
$$

$$
\varphi^{-1}\left(\int_{\Omega} \varphi \circ|x+y| d \mu\right) \leq \varphi^{-1}\left(\int_{\Omega} \varphi \circ|x| d \mu\right)+\varphi^{-1}\left(\int_{\Omega} \varphi \circ|y| d \mu\right)
$$

for every $x$ and $y$ belonging to the linear space of all the $\mu$-integrable step functions.

This is a weaker version of the classical result which asserts that inequality (1) holds for all $x$ and $y$ from the Banach space $L^{p}(\Omega, \Sigma, \mu)$. But on the one hand the classical result is a simple consequence of the above implication, on the other hand the weaker version is more suitable for our purposes.

In this paper we give conditions under which the converse implication holds. It follows from Mulholland's inequality [5] (cf. also M. Kuczma [3, p. 201, Theorem 1]) that, in general, the converse implication is false. (A simple example

Received by the editors September 26, 1988 and, in revised forms, February 4, 1989 and August 1, 1989; a partial result has been presented to the 26th ISFE, Sant Felieu de Guixols, Spain, April 24-May 3, 1988. The contents of this paper have been presented to the Northeast Conference on General Topology and Applications, New York, June 15-17, 1989.

1980 Mathematics Subject Classification (1985 Revision). Primary 46E30, 26D15, 26D10, 39C05; Secondary 39B40.

Key words and phrases. Measure space, Minkowski's inequality, subadditive functions, convex functions, normalized measure. 
and complementary comments are added at the end of this paper.) However our main result says that if the underlying measure space has two sets of positive finite measure such that one of them is of measure less than one and the other is of measure greater than one then the converse of Minkowski's inequality theorem is true.

Thanks to a recent theorem on subadditive bijections which is due to $T$. Swiatkowski and the present author [5] we prove the main result assuming that $\varphi:[0, \infty) \rightarrow[0, \infty)$ is bijective and right continuous at 0 .

A similar result is true when the sign of inequality (1) is reversed. In this case we do not even need any regularity condition on $\varphi$. This is due to the fact that now $\varphi^{-1}$ has to be a superadditive bijection of $\mathbf{R}_{+}$(then $\varphi^{-1}$ is increasing and, consequently, continuous).

Mulholland's inequality shows that the assumption on the existence of a set $A \in \Sigma$ such that $0<\mu(A)<1$ is essential for the above-mentioned theorems. To show that the remaining assumption on the existence of a set $B \in \Sigma$ such that $1<\mu(B)<\infty$ is also necessary, we prove the following generalization of Minkowski's inequality theorem:

If $(\Omega, \Sigma, \mu)$ is a nontrivial normalized measure space and $\varphi: \mathbf{R}_{+} \rightarrow \mathbf{R}_{+}$is a diffeomorphism of $\mathbf{R}_{+}$such that $\varphi^{\prime \prime}>0$ in $(0, \infty)$ and $\varphi^{\prime} / \varphi^{\prime \prime}$ is superadditive in $(0, \infty)$, then inequality (1) is true.

This is a complementary result to Mulholland's inequality, which corresponds to the case of counting measure.

A suitable generalization for the reversed inequality is also presented.

\section{Preliminaries AND AUXiliary Results}

By $\mathbf{N}, \mathbf{Z}, \mathbf{Q}, \mathbf{R}, \mathbf{R}_{+}$we denote positive integers, integers, rationals, reals, and nonnegative reals, respectively.

Let $(\Omega, \Sigma, \mu)$ be a measure space. By $S(\Omega, \Sigma, \mu)$ denote the linear space of all the $\mu$-integrable step functions $x: \Omega \rightarrow \mathbf{R}$ and by $S_{+}(\Omega, \Sigma, \mu)$ the set of all nonnegative $x \in S(\Omega, \Sigma, \mu)$. For the characteristic function of a set $A \subset \Omega$ we write $\chi_{A}$.

Let $\varphi: \mathbf{R}_{+} \rightarrow \mathbf{R}_{+}$. Then for every $x \in S(\Omega, \Sigma, \mu)$ there exist disjoint sets $A_{1}, \ldots, A_{n} \in \Sigma$ and $x_{1}, \ldots, x_{n} \in \mathbf{R}$ such that $x=\sum_{i=1}^{n} x_{i} \chi_{A_{i}}$ and

$$
\varphi \circ|x|=\sum_{i=1}^{n} \varphi\left(\left|x_{i}\right|\right) \chi_{A_{i}}+\varphi(0) \chi_{\Omega-\left(A_{1} \cup \cdots \cup A_{n}\right)} .
$$

If moreover $\varphi(0)=0$ it follows that $\varphi \circ|x| \in S(\Omega, \Sigma, \mu)$. Hence we obtain the following:

Remark 1. If $\varphi: \mathbf{R}_{+} \rightarrow \mathbf{R}_{+}$is bijective (i.e., one-to-one and onto) and $\varphi(0)=0$ then the functional $\mathbf{P}_{\varphi}: S(\Omega, \Sigma, \mu) \rightarrow \mathbf{R}_{+}$given by the formula

$$
\mathbf{P}_{\varphi}(x):=\varphi^{-1}\left(\int_{\Omega} \varphi \circ|x| d \mu\right), \quad x \in S(\Omega, \Sigma, \mu),
$$


is well defined and

$$
\mathbf{P}_{\varphi}(x)=\varphi^{-1}\left(\sum_{i=1}^{n} \mu\left(A_{i}\right) \varphi\left(\left|x_{i}\right|\right)\right), \quad x=\sum_{i=1}^{n} x_{i} \chi_{A_{i}} .
$$

In proving the main result we need some properties of subadditive functions. We quote the following:

Lemma 1. Suppose that $\psi: \mathbf{R}_{+} \rightarrow \mathbf{R}_{+}$is one-to-one, onto, and right continuous at 0 . If $\psi$ is subadditive, i.e.,

$$
\psi(s+t) \leq \psi(s)+\psi(t), \quad s, t \in \mathbf{R}_{+},
$$

then $\psi$ is a homeomorphism of $\mathbf{R}_{+}$. (In particular $\psi$ is increasing.)

Lemma 1 is the main result of [4].

Lemma 2. Suppose that $\psi, \varphi: \mathbf{R}_{+} \rightarrow \mathbf{R}_{+}$are subadditive. Then

(a) If $\psi$ is increasing then the composition $\psi \circ \varphi$ is subadditive;

(b) If $\psi$ is a homeomorphism of $\mathbf{R}_{+}$then $\psi^{-1}$ is superadditive, i.e.,

$$
\psi^{-1}(s+t) \geq \psi^{-1}(s)+\psi^{-1}(t), \quad s, t \geq 0 ;
$$

(c) If $\psi$ is a homeomorphism of $\mathbf{R}_{+}$and for some positive integer $n$, the $n$th iterate of $\psi, \psi^{n}$, is additive, then $\psi$ is additive and $\psi(t)=\psi(1) t$, $t \geq 0$.

Proof. Part (a) is trivial. (b) follows immediately from the fact that every homeomorphism of $\mathbf{R}_{+}$is increasing. To prove (c) note that, in view of (a), $\psi^{k}$ is subadditive for every $k \in \mathbf{N}$. From the assumption we have $\psi^{n}(t)=c t$ for some $c>0$ and all $t \geq 0$. Thus $\psi^{n-1}(t)=c \psi^{-1}(t)$ and by (b) $\psi$ is additive.

Remark 2. In (b) the assumption that the domain of $\psi$ is $\mathbf{R}_{+}$is essential as is shown by the following example. The function $\psi(t)=1 / t \quad(t>0)$, is a subadditive homeomorphism of $(0, \infty)$ and $\psi^{-1}$, being equal to $\psi$, is also subadditive.

By an easy induction we obtain the following:

Lemma 3. Supose that $\varphi: \mathbf{R}_{+} \rightarrow \mathbf{R}_{+}$is a homeomorphism of $\mathbf{R}_{+}$. If

$$
\varphi^{-1}\left(\varphi\left(x_{1}+y_{1}\right)+\varphi\left(x_{2}+y_{2}\right)\right) \leq \varphi^{-1}\left(\varphi\left(x_{1}\right)+\varphi\left(x_{2}\right)\right)+\varphi^{-1}\left(\varphi\left(y_{1}\right)+\varphi\left(y_{2}\right)\right)
$$

for all nonnegative $x_{1}, x_{2}, y_{1}, y_{2}$ then

$$
\begin{aligned}
\varphi^{-1}\left(\varphi\left(x_{1}+y_{1}\right)+\cdots+\varphi\left(x_{n}+y_{n}\right)\right) \leq & \varphi^{-1}\left(\varphi\left(x_{1}\right)+\cdots+\varphi\left(x_{n}\right)\right) \\
& +\varphi^{-1}\left(\varphi\left(y_{1}\right)+\cdots+\varphi\left(y_{n}\right)\right)
\end{aligned}
$$

for all $n \in \mathbf{N}$ and nonnegative $x_{1}, \ldots, x_{n} ; y_{1}, \ldots, y_{n}$.

From a well-known theorem of Kronecker it follows that if $a, b \in \mathbf{R}$ are not commensurable (i.e., $b / a \notin \mathbf{Q}$ and $a \neq 0$ ) then the set

$$
D:=\{n a+m b: n, m \in \mathbf{Z}\}
$$

is dense in $\mathbf{R}$. 
In this paper we need the following:

Lemma 4. If $a, b \in \mathbf{R}, a<0<b$ and $b / a$ is irrational then the set

$$
A:=\{n a+m b: n, m \in \mathbf{N}\}
$$

is dense in $\mathbf{R}$.

Proof. For $X \subset \mathbf{R}$ let $-X:=\{-x: x \in X\}$. Put

$$
B:=\{n a-m b: n, m \in \mathbf{N} \cup\{0\}\} .
$$

We evidently have $D=A \cup(-A) \cup B \cup(-B)$. Since $B \cup(-B)$ has no accumulation point in $\mathbf{R}$, it follows from Kronecker's theorem that the set $A \cup(-A)$ is dense in $\mathbf{R}$. In particular 0 is a cluster point of this set. Therefore it is clear that there exists a sequence

$$
n_{k} a+m_{k} b \in A \quad\left(k, n_{k}, m_{k} \in \mathbf{N}\right),
$$

such that

$$
\lim _{k \rightarrow \infty}\left(n_{k} a+m_{k} b\right)=0 .
$$

This implies that

$$
\lim _{k \rightarrow \infty} n_{k}=\lim _{k \rightarrow \infty} m_{k}=\infty .
$$

Take now $t \in \mathbf{R}$ and $\varepsilon>0$. It follows from the density of the set $D$ that there exist $n, m \in \mathbf{Z}$ such that

$$
|n a+m b-t|<\varepsilon / 2 .
$$

Now choose a $k_{0}$ such that for $k \geq k_{0}$ we have

$$
\left|n_{k} a+m_{k} b\right|<\varepsilon / 2, \quad n_{k}>|n|, \quad m_{k}>|m| .
$$

Since $n_{k}+n$ and $m_{k}+m$ are positive integers, we have

$$
\left(n_{k}+n\right) a+\left(m_{k}+m\right) b \in A .
$$

Moreover, from (4) and (5) it follows that

$$
\left|\left(n_{k}+n\right) a+\left(m_{k}+m\right) b-t\right|<\varepsilon,
$$

which completes the proof of the lemma.

Remark 3. From the identity $a^{n} b^{m}=e^{n \log a+m \log b}$ we get the following multiplicative counterpart of Lemma 4: If $0<a<1<b$ and $\log a$ and $\log b$ are not commensurable, then the set

$$
M:=\left\{a^{n} b^{m}: n, m \in \mathbf{N}\right\}
$$

is dense in the interval $(0, \infty)$. 


\section{THE MAIN RESULT}

In this section we prove the following:

Theorem 1. Let $(\Omega, \Sigma, \mu)$ be a measure space with two sets $A, B \in \Sigma$ such that

$$
0<\mu(A)<1<\mu(B)<\infty .
$$

Suppose that $\varphi: \mathbf{R}_{+} \rightarrow \mathbf{R}_{+}$is a bijection such that $\varphi^{-1}$ is continuous at 0 and $\varphi(0)=0$. If the functional $\mathbf{P}_{\varphi}: S_{+}(\Omega, \Sigma, \mu) \rightarrow \mathbf{R}_{+}$defined by the formula

$$
\mathbf{P}_{\varphi}(x):=\varphi^{-1}\left(\int_{\Omega} \varphi \circ x d \mu\right)
$$

satisfies the triangle inequality

$$
\mathbf{P}_{\varphi}(x+y) \leq \mathbf{P}_{\varphi}(x)+\mathbf{P}_{\varphi}(y), \quad x, y \in S_{+}(\Omega, \Sigma, \mu),
$$

then there exists a $p \geq 1$ such that $\varphi(t)=\varphi(1) t^{p}(t \geq 0)$.

Proof. Note that there are two distinct sets $C, D \in \Sigma$ of finite and positive measure. In fact, we may take $C:=A$ and $D:=B \backslash A$. Write $c:=\mu(C)$ and $d:=\mu(D)$. Taking in the triangle inequality (7),

$$
x:=x_{1} \chi_{C}+x_{2} \chi_{D}, \quad y:=y_{1} \chi_{C}+y_{2} \chi_{D}
$$

and making use of Remark 1 and formula (3), we get $\varphi^{-1}\left(c \varphi\left(x_{1}+y_{1}\right)+d \varphi\left(x_{2}+y_{2}\right)\right) \leq \varphi^{-1}\left(c \varphi\left(x_{1}\right)+d \varphi\left(x_{2}\right)\right)+\varphi^{-1}\left(c \varphi\left(y_{1}\right)+d \varphi\left(y_{2}\right)\right)$ for all nonnegative $x_{1}, x_{2}, y_{1}, y_{2}$. Substituting here

$$
y_{1}=x_{2}=0, \quad x_{1}=\varphi^{-1}(s / c), \quad y_{2}=\varphi^{-1}(t / d) \quad(s, t \geq 0),
$$

and taking into account that $\varphi(0)=0$, we obtain

$$
\varphi^{-1}(s+t) \leq \varphi^{-1}(s)+\varphi^{-1}(t) \quad(s, t \geq 0),
$$

i.e., that $\varphi^{-1}$ is subadditive in $\mathbf{R}_{+}$. Since it is continuous at 0 , it follows from Lemma 1 that $\varphi$ is an increasing homeomorphism of $\mathbf{R}_{+}$.

Let, for a moment, $C$ denote an arbitrary $\Sigma$-measurable set such that $0<$ $\mu(C)<\infty$ and put $c:=\mu(C)$. Setting in (7) the functions $x=s \chi_{C}, y=t \chi_{C}$, we obtain the inequality

$$
\varphi^{-1}(c \varphi(s+t)) \leq \varphi^{-1}(c \varphi(s))+\varphi^{-1}(c \varphi(t)) \quad(s, t \geq 0) .
$$

Thus the function $\psi:=\varphi^{-1} \circ(c \varphi)$ is a subadditive homeomorphism of $\mathbf{R}_{+}$. In view of Lemma $2 \mathrm{a}$, the $n$th iterate of $\psi$, the function $\psi^{n}=\varphi^{-1} \circ\left(c^{n} \varphi\right)$ is also subadditive for every $n \in \mathbf{N}$. Writing

$$
a:=\mu(A), \quad b:=\mu(B)
$$

we hence conclude that the functions $\varphi^{-1} \circ\left(a^{n} \varphi\right), \varphi^{-1} \circ\left(b^{m} \varphi\right)$ and, consequently, $\varphi^{-1} \circ\left(a^{n} b^{m} \varphi\right)$ are subadditive homeomorphisms of $\mathbf{R}_{+}$for all $n, m \in \mathbf{N}$. 
Now we consider two cases.

Case $1^{\circ}$. There exist $A, B \in \Sigma$ satisfying (6) and such that

$$
\frac{\log b}{\log a} \notin \mathbf{Q}
$$

i.e., that $\log a$ and $\log b$ are incommensurable.

In this case, in view of Lemma 4 (cf. Remark 3), the set

$$
M:=\left\{a^{n} b^{m}: n, m \in \mathbf{N}\right\}
$$

is dense in $\mathbf{R}_{+}$. Therefore it easily follows, from the subadditivity of $\varphi^{-1} \circ$ $\left(a^{n} b^{m} \varphi\right)$ for all $n, m \in \mathbf{N}$ and from the continuity of $\varphi^{-1}$, that for each $r>0$, the function $\varphi^{-1} \circ(r \varphi)$ is subadditive. Since it is an increasing homeomorphism of $\mathbf{R}_{+}$, by Lemma $2 b$ we infer that its inverse, $\varphi^{-1} \circ\left(r^{-1} \varphi\right)$, is superadditive. Consequently, the function $\varphi^{-1} \circ(r \varphi)$ is additive for every $r>0$. Hence, for every $r>0$ there exists a positive number $m(r)$ such that

$$
\varphi^{-1}(r \varphi(t))=m(r) t \quad(r>0, t \geq 0) .
$$

Setting here $t=1$ we get $m(r)=\varphi^{-1}(\varphi(1) r)$ for $r>0$, which implies that the function $m:(0, \infty) \rightarrow(0, \infty)$ is continuous. Moreover for $r_{1}, r_{2}>0$ we have

$$
\varphi^{-1}\left(r_{1} \varphi(t)\right)=m\left(r_{1}\right) t, \quad \varphi^{-1}\left(r_{2} \varphi(t)\right)=m\left(r_{2}\right) t \quad(t \geq 0) .
$$

Composing the functions occuring on the left and on the right sides, respectively, we obtain

$$
\varphi^{-1}\left(r_{1} r_{2} \varphi(t)\right)=m\left(r_{1}\right) m\left(r_{2}\right) t \quad(t \geq 0) .
$$

On the other hand, from (7), we get

$$
\varphi^{-1}\left(r_{1} r_{2} \varphi(t)\right)=m\left(r_{1} r_{2}\right) t \quad(t \geq 0) .
$$

The last two equations show that

$$
m\left(r_{1} r_{2}\right)=m\left(r_{1}\right) m\left(r_{2}\right) \quad\left(r_{1}, r_{2}>0\right) .
$$

Thus $m$ is a continuous solution of the multiplicative Cauchy equation. This implies that (cf. J. Aczel [1, p. 41]) $m(r)=r^{1 / p}$ for some $p \in \mathbf{R}, p \neq 0$, and for all $r>0$. Since $m(r)=\varphi^{-1}(\varphi(1) r)$, we hence get

$$
\varphi^{-1}(\varphi(1) r)=r^{1 / p} \quad(r>0),
$$

or, equivalently,

$$
\varphi(r)=\varphi(1) r^{p} \quad(r>0) .
$$

Note that $p$ has to be positive as $\varphi$ is increasing. The inequality $p \geq 1$ is a simple consequence of the triangle inequality (7). This completes the proof in case $1^{\circ}$.

Now consider the remaining case:

Case $2^{\circ}$. For every $C, D \in \Sigma$ such that $0<\mu(C)<1<\mu(D)<\infty$ we have

$$
\frac{\log d}{\log c} \in \mathbf{Q}, \quad \text { where } c:=\mu(c), d:=\mu(D),
$$

i.e., $\log c$ and $\log d$ are commensurable. 
First observe that in this case the following implication holds:

$$
(C \in \Sigma, 0<c:=\mu(C)<\infty) \Rightarrow \varphi^{-1} \circ(c \varphi) \text { is additive. }
$$

It is evidently true for $c=1$. Suppose that $0<c<1$. Since $b=\mu(B)>1$, it follows from $2^{\circ}$ that there exist $n, m \in \mathbf{N}$ such that $\log b / \log c=-n / m$ or, equivalently, that

$$
c^{n} b^{m}=1 \text {. }
$$

According to the first part of the proof the functions $\varphi^{-1} \circ\left(c^{n} \varphi\right)$ and $\varphi^{-1} \circ\left(b^{m} \varphi\right)$ are subadditive. But from (10) it follows that these functions are inverses of one another and, being increasing, they have to be additive (cf. Lemma $2 b$ ).

Suppose that $c>1$. Then from $2^{\circ}$ we have $\log c / \log a=-n / m$ for some positive integers $n, m$ and $a=\mu(A)<1$, which allows us to argue in the same manner as in the previous case. This proves (9).

Let $C:=B \backslash A$. From (9) it follows that there are $\alpha, \gamma>0$ such that

$$
\varphi^{-1}(a \varphi(t))=\alpha t, \quad \varphi^{-1}(c \varphi(t))=\gamma t \quad(t \geq 0),
$$

which means that $\varphi$ satisfies the following system of functional equations:

$$
a \varphi(t)=\varphi(\alpha t), \quad c \varphi(t)=\varphi(\gamma t) \quad(t \geq 0) .
$$

Since $A$ and $C$ are disjoint, taking in the triangle inequality (7)

$$
x=s \chi_{A}+\bar{s} \chi_{C}, \quad y=t \chi_{A}+\bar{t} \chi_{C} \in S_{+}(\Omega, \Sigma, \mu),
$$

we obtain

$$
\varphi^{-1}(a \varphi(s+t)+c \varphi(\bar{s}+\bar{t})) \leq \varphi^{-1}(a \varphi(s)+c \varphi(\bar{s}))+\varphi^{-1}(a \varphi(t)+c \varphi(\bar{t})) .
$$

Applying (11) we get

$$
\varphi^{-1}(\varphi(\alpha s+\alpha t)+\varphi(\gamma \bar{s}+\gamma \bar{t})) \leq \varphi^{-1}(\varphi(\alpha s)+\varphi(\gamma \bar{s}))+\varphi^{-1}(\varphi(\alpha t)+\varphi(\gamma \bar{t}))
$$

for all nonnegative $s, \bar{s}, t, \bar{t}$. Setting here

$$
s:=\frac{x_{1}}{\alpha}, \quad \bar{s}:=\frac{x_{2}}{\gamma}, \quad t:=\frac{y_{1}}{\alpha}, \quad \bar{t}:=\frac{y_{2}}{\gamma},
$$

we get

$$
\varphi^{-1}\left(\varphi\left(x_{1}+y_{1}\right)+\varphi\left(x_{2}+y_{2}\right)\right) \leq \varphi^{-1}\left(\varphi\left(x_{1}\right)+\varphi\left(x_{2}\right)\right)+\varphi^{-1}\left(\varphi\left(y_{1}\right)+\varphi\left(y_{2}\right)\right)
$$

for all nonnegative $x_{1}, x_{2}, y_{1}, y_{2}$. Now it follows from Lemma 3 that for each $k \in \mathbf{N}$ and for all nonnegative $x_{1}, \ldots, x_{k} ; y_{1}, \ldots, y_{k}$,

$$
\varphi^{-1}\left(\sum_{i=1}^{k} \varphi\left(x_{i}+y_{i}\right)\right) \leq \varphi^{-1}\left(\sum_{i=1}^{k} \varphi\left(x_{i}\right)\right)+\varphi^{-1}\left(\sum_{i=1}^{k} \varphi\left(y_{i}\right)\right) .
$$

Setting $x_{1}=\cdots=x_{k}:=s, y_{1}=\cdots=y_{k}:=t, s, t \geq 0$, we obtain

$$
\varphi^{-1}(k \varphi(s+t)) \leq \varphi^{-1}(k \varphi(s))+\varphi^{-1}(k \varphi(t)) \quad(s, t \geq 0),
$$


which means that the function $\varphi^{-1} \circ(k \varphi)$ is subadditive for all $k \in \mathbf{N}$. According to the first part of the proof, the function $\varphi^{-1} \circ\left(a^{n} \varphi\right) \quad(n \in \mathbf{N})$ is subadditive (actually, in view of (9), it is additive). Therefore, by Lemma 2a, all the functions

$$
\varphi^{-1} \circ\left(k a^{n} \varphi\right) \quad(k, n \in \mathbf{N}),
$$

are subadditive. Since $0<a<1$, the set

$$
\left\{k a^{n}: k, n \in \mathbf{N}\right\}
$$

is dense in $\mathbf{R}_{+}$.

Now we can repeat the last half of the argument which we used in Case $1^{\circ}$. This ends the proof.

Remark 4. Suppose that the functional $\mathbf{P}_{\varphi}$ satisfies the reversed inequality. Then $\varphi^{-1}$ is superadditive. One can easily verify that this function is an increasing homeomorphism of $\mathbf{R}_{+}$and that Lemma 3 for the reversed inequality remains true. Therefore, in the same way as Theorem 1, we can prove the following:

Theorem 2. Let $(\Omega, \Sigma, \mu)$ be a measure space with two sets $A, B \in \Sigma$ such that $0<\mu(A)<1<\mu(B)<\infty$. Suppose that $\varphi: \mathbf{R}_{+} \rightarrow \mathbf{R}_{+}$is an arbitrary bijection of $\mathbf{R}_{+}$such that $\varphi(0)=0$. If the functional $\mathbf{P}_{\varphi}: S_{+}(\Omega, \Sigma, \mu) \rightarrow \mathbf{R}_{+}$defined by the formula

$$
\mathbf{P}_{\varphi}(x):=\varphi^{-1}\left(\int_{\Omega} \varphi \circ x d \mu\right)
$$

satisfies the inequality $\mathbf{P}_{\varphi}(x+y) \geq \mathbf{P}_{\varphi}(x)+\mathbf{P}_{\varphi}(y) ;, x, y \in S_{+}(\Omega, \Sigma, \mu)$, then there exists a $p, 0<p \leq 1$, such that $\varphi(t)=\varphi(1) t^{p}(t \geq 0)$.

\section{DisCUSSION OF THE ASSUMPTIONS AND A GENERALIZATION OF MINKOWSKI'S INEQUALITY FOR PROBABILITY SPACE}

The assumption on the existence of two sets $A, B \in \Sigma$ such that $0<\mu(A)<$ $1<\mu(B)<\infty$ plays a key role in the proof of Theorem 1 . To see that the existence of $A \in \Sigma$ such that $0<\mu(A)<1$ is essential, consider the following:

Example. Let $(\Omega, \Sigma, \mu)$ be a measure space with $\Omega=\{a, b\}, \Sigma=2^{\Omega}$ and $\mu(\{a\})=\mu(\{b\})=1$. We have $\mu(\Sigma)=\{0,1,2\}$; thus, there is no $A \in \Sigma$ such that $0<\mu(A)<1$. Since each $x \in S_{+}(\Omega, \Sigma, \mu)$ is of the form $x=$ $x_{1} \chi_{\{a\}}+x_{2} \chi_{\{b\}}$, the triangle inequality (7) reduces to the inequality

$$
\varphi^{-1}\left(\varphi\left(x_{1}+y_{1}\right)+\varphi\left(x_{2}+y_{2}\right)\right) \leq \varphi^{-1}\left(\varphi\left(x_{1}\right)+\varphi\left(x_{2}\right)\right)+\varphi^{-1}\left(\varphi\left(y_{1}\right)+\varphi\left(y_{2}\right)\right)
$$

for all nonnegative $x_{1}, x_{2}, y_{1}, y_{2}$. Note that the function $\varphi(t)=e^{t}-1 \quad(t \geq 0)$ satisfies this inequality. In fact, (12) becomes now

$$
\log \left(e^{x_{1}+y_{1}}+e^{x_{2}+y_{2}}-1\right) \leq \log \left(e^{x_{1}}+e^{x_{2}}-1\right)+\log \left(e^{y_{1}}+e^{y_{2}}-1\right)
$$


which, after simple calculations, may be written in the form

$$
\left(e^{x_{1}}-1\right)\left(e^{y_{2}}-1\right)+\left(e^{x_{2}}-1\right)\left(e^{y_{1}}-1\right) \geq 0 .
$$

This is true for all nonnegative arguments. Thus we have shown that existence of $A \in \Sigma$ such that $0<\mu(A)<1$ is essential.

Let us mention here that H. P. Mulholland [6] found a whole class of nonpower functions which satisfy (12). Namely he proved the following:

Theorem. If $\varphi: \mathbf{R}_{+} \rightarrow \mathbf{R}_{+}$is an increasing and convex bijection of $\mathbf{R}_{+}$such that the function $\varphi(t):=\log \varphi\left(e^{t}\right) \quad(t \in \mathbf{R})$ is convex in $\mathbf{R}$, then for all $n \in \mathbf{N}$ and nonnegative $x_{1}, \ldots, x_{n} ; y_{1}, \ldots, y_{n}$,

$$
\varphi^{-1}\left(\sum_{i=1}^{n} \varphi\left(x_{i}+y_{i}\right)\right) \leq \varphi^{-1}\left(\sum_{i=1}^{n} \varphi\left(x_{i}\right)\right)+\varphi^{-1}\left(\sum_{i=1}^{n} \varphi\left(y_{i}\right)\right) .
$$

Remark 5. A not easy proof of this theorem (cf. M. Kuczma [3, p. 198-201]) may be simplified a little by means of our Lemma 3. Namely, from Lemma 3 it follows that it is sufficient to prove (13), only for $n=2$.

With respect to the role played by the normalized measures in probability the remaining question, whether the existence of the set $\beta \in \mathbf{Z}$ such that $1<$ $\mu(B)<\infty$ is essential, seems to be of interest. It appears that the answer is "yes" and it follows from the following:

Theorem 3. Let $(\Omega, \Sigma, \mu)$ be a normalized measure space with at least one set $A \in \Sigma$ such that $0<\mu(A)<1$. Suppose that $\varphi: \mathbf{R}_{+} \rightarrow \mathbf{R}_{+}$is a homeomorphism of $\mathbf{R}_{+}$. Then:

$1^{\circ}$. The functional $\mathbf{P}_{\varphi}$ satisfies the triangle inequality (7) for all $x, y \in$ $S_{+}(\Omega, \Sigma, \mu)$ if and only if the function $f: \mathbf{R}_{+}^{2} \rightarrow \mathbf{R}_{+}$defined by the formula

$$
f(s):=\varphi\left(\varphi^{-1}\left(s_{1}\right)+\varphi^{-1}\left(s_{2}\right)\right) \quad\left(s:=\left(s_{1}, s_{2}\right)\right),
$$

is concave in $\mathbf{R}_{+}^{2}$.

$2^{\circ}$. If $\varphi$ is twice continuously differentiable, $\varphi^{\prime \prime}>0$ in $(0, \infty)$ and the function $\varphi^{\prime} / \varphi^{\prime \prime}$ is superadditive in $(0, \infty)$, then the function $f$ is concave in $\mathbf{R}_{+}^{2}$.

Proof. $1^{\circ}$. Suppose that $\mathbf{P}_{\varphi}$ satisfies (7) and put $B:=\Omega \backslash A, a:=\mu(A)$, $b:=\mu(B)$. By assumption we have $a+b=1$ and $0<a<1$. Setting in (7): $x=x_{1} \chi_{A}+x_{2} \chi_{B}, y=y_{1} \chi_{A}+y_{2} \chi_{B} \in S_{+}(\Omega, \Sigma, \mu)$, we get $\varphi^{-1}\left(a \varphi\left(x_{1}+y_{1}\right)+b \varphi\left(x_{2}+y_{2}\right)\right) \leq \varphi^{-1}\left(a \varphi\left(x_{1}\right)+b \varphi\left(x_{2}\right)\right)+\varphi^{-1}\left(a \varphi\left(y_{1}\right)+b \varphi\left(y_{2}\right)\right)$ and, as $\varphi$ is increasing, $a \varphi\left(x_{1}+y_{1}\right)+b \varphi\left(x_{2}+y_{2}\right) \leq \varphi\left[\varphi^{-1}\left(a \varphi\left(x_{1}\right)+b \varphi\left(x_{2}\right)\right)+\varphi^{-1}\left(a \varphi\left(y_{1}\right)+b \varphi\left(y_{2}\right)\right)\right]$.

Substituting here

$$
x_{1}:=\varphi^{-1}\left(s_{1}\right), \quad x_{2}:=\varphi^{-1}\left(t_{1}\right), \quad y_{1}:=\varphi^{-1}\left(s_{2}\right), \quad y_{2}:=\varphi^{-1}\left(t_{2}\right)
$$


$\left(s_{1}, s_{2}, t_{1}, t_{2} \geq 0\right)$, we obtain

$$
\begin{gathered}
a \varphi\left(\varphi^{-1}\left(s_{1}\right)+\varphi^{-1}\left(s_{2}\right)\right)+b \varphi\left(\varphi^{-1}\left(t_{1}\right)+\varphi^{-1}\left(t_{2}\right)\right) \\
\leq \varphi\left[\varphi^{-1}\left(a s_{1}+b s_{2}\right)+\varphi^{-1}\left(a t_{1}+b t_{2}\right)\right],
\end{gathered}
$$

which may be written in the form

$$
a f(s)+b f(t) \leq f(a s+b t), \quad s:=\left(s_{1}, s_{2}\right), t:=\left(t_{1}, t_{2}\right) .
$$

hence, using only induction, one can show that

$$
\alpha_{n, j} f(s)+\left(1-\alpha_{n, j}\right) f(t) \leq f\left(\alpha_{n, j} s+\left(1-\alpha_{n, j}\right) t\right)
$$

where

$$
\alpha_{n, j}:=\sum_{k=0}^{j}\left(\begin{array}{l}
n \\
k
\end{array}\right) a^{n-k} b^{k} \quad(n \in \mathbf{N} ; j=0,1, \ldots, n) .
$$

Since the set of all $\alpha_{n, j}$ is dense in $(0,1)$ it follows from the continuity of $f$ that $f$ is concave (for another argument, cf. [4]).

To prove the converse implication take $x, y \in S_{+}(\Omega, \Sigma, \mu)$. There exist disjoint sets $A_{1}, \ldots, A_{n} \in \Sigma$ such that

$$
x=\sum_{i=1}^{n} x_{i} \chi_{A_{i}}, \quad y=\sum_{i=1}^{n} y_{i} \chi_{A_{i}},
$$

where $x_{i}, y_{i}$ are nonnegative. Let $a_{i}:=\mu\left(A_{i}\right), i=1, \ldots, n$. Since $a_{1}+\cdots+$ $a_{n}=1$, from the concavity of $f$ it follows that

$$
\sum_{i=1}^{n} a_{i} f\left(s_{i}, t_{i}\right) \leq f\left(\sum_{i=1}^{n} a_{i} s_{i}, \sum_{i=1}^{n} a_{i} t_{i}\right)
$$

for all nonnegative $s_{1}, \ldots, s_{n} ; t_{1}, \ldots, t_{n}$. According to the definition of the function $f$, we hence get

$$
\sum_{i=1}^{n} a_{i} \varphi\left(\varphi^{-1}\left(s_{i}\right)+\varphi^{-1}\left(t_{i}\right)\right) \leq \varphi\left(\varphi^{-1}\left(\sum_{i=1}^{n} a_{i} s_{i}\right)+\varphi^{-1}\left(\sum_{i=1}^{n} a_{i} t_{i}\right)\right) .
$$

Substituting $s_{i}:=\varphi\left(x_{i}\right), t_{i}:=\varphi\left(y_{i}\right) \quad(i=1, \ldots, n)$, we hence obtain

$$
\varphi^{-1}\left(\sum_{i=1}^{n} a_{i} \varphi\left(x_{i}+y_{i}\right)\right) \leq \varphi^{-1}\left(\sum_{i=1}^{n} a_{i} \varphi\left(x_{i}\right)\right)+\varphi^{-1}\left(\sum_{i=1}^{n} a_{i} \varphi\left(y_{i}\right)\right)
$$

or, equivalently,

$$
\varphi^{-1}\left(\int_{\Omega} \varphi \circ|x+y| d \mu\right) \leq \varphi^{-1}\left(\int_{\Omega} \varphi \circ|x| d \mu\right)+\varphi^{-1}\left(\int_{\Omega} \varphi \circ|y| d \mu\right)
$$

which was to be shown. This completes the proof of $1^{\circ}$.

$2^{\circ}$. Put, for convenience,

$$
h:=\varphi^{-1} .
$$


Then

$$
f(s, t)=h^{-1}(h(s)+h(t)) \quad(s, t \geq 0)
$$

and, since

$$
\varphi^{\prime}(t)=\frac{1}{h^{\prime}(\varphi(t))}, \quad \varphi^{\prime \prime}(t)=-\frac{h^{\prime \prime}(\varphi(t))}{\left(h^{\prime}(\varphi(t))^{3}\right.} \quad(t>0)
$$

we have

$$
h^{\prime}>0, \quad h^{\prime \prime}<0
$$

and

$$
\frac{\varphi^{\prime}}{\varphi^{\prime \prime}}=-\frac{\left(h^{\prime}\right)^{2}}{h^{\prime \prime}} \circ \varphi
$$

Consequently, the function

$$
\psi:=\frac{h^{\prime 2}}{h^{\prime \prime}} \circ \varphi \quad \text { is subadditive in }(0, \infty) .
$$

Denote (for this part of proof only) by $x, y$ arbitrary positive reals and let $u, v \in \mathbf{R}$. The function

$$
g(t):=f(x+u t, y+v t)
$$

is well defined and of the class $C^{2}$ in a neighbourhood of 0 . To prove that $f$ is concave in $\mathbf{R}_{+}^{2}$ it suffices to show that for all $x, y \in(0, \infty)$ and $u, v \in \mathbf{R}$ we have $g^{\prime \prime}(0) \leq 0$ (cf. G. H. Hardy, J. E. Littlewood, and G. Pólya [2, 76-81]). From the definition of $g$ and from (15), we have

$$
h(g(t))=h(x+u t)+h(y+v t)
$$

for $t$ in a neighborhood of 0 . Hence we get

$$
\begin{aligned}
& h^{\prime}(g(0)) g^{\prime}(0)=h^{\prime}(x) u+h^{\prime}(y) v, \\
& h^{\prime \prime}(g(0))\left(g^{\prime}(0)\right)^{2}+h^{\prime}(g(0)) g^{\prime \prime}(0)=h^{\prime \prime}(x) u^{2}+h^{\prime \prime}(y) v^{2} .
\end{aligned}
$$

Eliminating from these two equations $g^{\prime}(0)$, we obtain

$$
\left(h^{\prime}(g(0))\right)^{3} g^{\prime \prime}(0)=F u^{2}-2 G u v+H v^{2}
$$

where

$$
\begin{aligned}
& F:=h^{\prime \prime}(x)\left(h^{\prime}(g(0))\right)^{2}-h^{\prime \prime}(g(0))\left(h^{\prime}(x)\right)^{2}, \\
& G:=h^{\prime \prime}(g(0)) h^{\prime}(x) h^{\prime}(y), \\
& H:=h^{\prime \prime}(y)\left(h^{\prime}(g(0))\right)^{2}-h^{\prime \prime}(g(0))\left(h^{\prime}(y)\right)^{2} .
\end{aligned}
$$

From (16) and (18) we infer that $g^{\prime \prime}(0) \leq 0$ if

$$
F \leq 0, \quad H \leq 0, \text { and } F H-G^{2} \geq 0 .
$$


Note that all these conditions are fulfilled. In fact, in view of (16), the function

$$
\psi=\frac{\left(h^{\prime}\right)^{2}}{h^{\prime \prime}} \circ \varphi
$$

has negative values. Since, by $(17), \psi$ is subadditive, it follows that it is decreasing in $(0, \infty)$ and, evidently, so is the function $\psi \circ \varphi^{-1}=\left(h^{\prime}\right)^{2} / h^{\prime \prime}$. Since $x<g(0)=h^{-1}(h(x)+h(y))$, we hence obtain

$$
\frac{\left(h^{\prime}(g(0))\right)^{2}}{h^{\prime \prime}(g(0))}<\frac{\left(h^{\prime}(x)\right)^{2}}{h^{\prime \prime}(x)},
$$

which implies that $F<0$. In the same way we may show that $H<0$.

Now from the definitions of $F, G, H$ we have

$$
\begin{aligned}
& \frac{F H-G^{2}}{h^{\prime \prime}(x) h^{\prime \prime}(y) h^{\prime \prime}(g(0))} \\
& =\left(h^{\prime}(g(0))\right)^{2}\left[\frac{\left(h^{\prime}(g(0))\right)^{2}}{h^{\prime \prime}(g(0))}-\frac{\left(h^{\prime}(x)\right)^{2}}{h^{\prime \prime}(x)}-\frac{\left(h^{\prime}(y)\right)^{2}}{h^{\prime \prime}(y)}\right] .
\end{aligned}
$$

It follows that $F H-G^{2} \geq 0$ iff

$$
\frac{\left(h^{\prime}(g(0))\right)^{2}}{h^{\prime \prime}(g(0))} \leq \frac{\left(h^{\prime}(x)\right)^{2}}{h^{\prime \prime}(x)}+\frac{\left(h^{\prime}(y)\right)^{2}}{h^{\prime \prime}(y)} .
$$

Substituting here $x=\varphi(s), y=\varphi(t)$ and taking into account that $g(0)=$ $\varphi\left(\varphi^{-1}(x)+\varphi^{-1}(y)\right)$, we obtain $\psi(s+t) \leq \psi(s)+\psi(t)$. Thus the inequality $F H-G^{2} \geq 0$ is equivalent to the subadditivity of $\psi$. This completes the proof.

Modifying in an obvious way the proof of Theorem 3 we can prove the following:

Theorem 4. Let $(\Omega, \Sigma, \mu)$ be a normalized measure space with at least one set $A \in \Sigma$ such that $0<\mu(A)<1$. Suppose that $\varphi: \mathbf{R}_{+} \rightarrow \mathbf{R}_{+}$is a homeomorphism of $\mathbf{R}_{+}$. Then

$1^{\circ}$. The functional $\mathbf{P}_{\varphi}$ satisfies the inequality

$$
\mathbf{P}_{\varphi}(x+y) \geq \mathbf{P}_{\varphi}(x)+\mathbf{P}_{\varphi}(y), \quad x, y \in S_{+}(\Omega, \Sigma, \mu),
$$

if and only if the function $f$ defined by formula (14) is convex.

$2^{\circ}$. If $\varphi$ is twice continuously differentiable, $\varphi^{\prime \prime}<0$ in $(0, \infty)$ and the function $\varphi^{\prime} / \varphi^{\prime \prime}$ is subadditive in $(0, \infty)$, then the function $f$ is convex.

Remark 6. If $\varphi: \mathbf{R}_{+} \rightarrow \mathbf{R}_{+}$is a diffeomorphism of the class $C^{2}$ such that the function $\varphi^{\prime} / \varphi^{\prime \prime}$ is additive (i.e., both subadditive and superadditive) then there exists a $p \in \mathbf{R}$ such that

$$
\frac{\varphi^{\prime}(t)}{\varphi^{\prime \prime}(t)}=\frac{1}{p-1} t .
$$

Since $\varphi(0)=0$ we hence obtain that $\varphi(t)=c t^{p} \quad(t>0)$ for some $c>0$.

Example. One can easily verify that the functions $\varphi(t)=t \exp (-1 / t)$ for $t>0$, $\varphi(0)=0$, and $\varphi(t)=t^{2} /(t+1), t \geq 0$, satisfy all the conditions of Theorem $3.2^{\circ}$. 


\section{REFERENCES}

1. J. Aczél, Lectures on functional equations and their applications, Academic Press, New York and London, 1966.

2. G. H. Hardy, J. E. Littlewood, and G. Pólya, Inequalities, 2nd ed., Cambridge University Press, London and New York, 1952.

3. M. Kuczma, An introduction to the theory of functional equations and inequalities, Polish Scientific Publishers and Silesian University, Warszawa, Kraków, and Katowice, 1985.

4. N. Kuhn, A note on t-convex functions, General Inequalities 4, International Series of Numerical Mathematics, vol. 71, Birkhäuser Verlag, Basel, 1984, pp. 269-276.

5. J. Matkowski and T. Swiatkowski, Quasi-monotonicity, subadditive bijections of $\mathbf{R}_{+}$and characterization of $L^{p}$-norm, J. Math. Anal. and Appl. (to appear).

6. H. P. Mulholland, On generalizations of Minkowski's inequality in the form of a triangle inequality, Proc. London Math. Soc. 51 (1950), 294-307.

Department of Mathematics, Technical University, Findera 32, 43-300 Bielsko-Biala, POLAND 